InnOvaciOnes de NegOciOs 16(32): 232-270

(c) 2019 UANL, Impreso en México (ISSN: 2007-1191)

Recepción: 4 Marzo 2019 Aceptación: 25 Marzo 2019

\title{
La incorporación de la Industria 4.0 en el sector de autopartes en Nuevo León, México (The incorporation of industry 4.0 in the auto parts sector in Nuevo Leon, Mexico)
}

\author{
Karla María Nava Aguirre, Juan Gil Silva Ábrego, Alejandra Guajardo \\ García, Orlando Uriel Leyva Velázquez, Carol Yareslie Torres Camarillo \\ Universidad de Monterrey \\ karla.nava@udem.edu, juan.silva@udem.edu, alejandra.guajardog@udem.edu, \\ orlando.leyva@udem.edu, carol.torres@udem.edu
}

Abstract: Mexico is the fifth producer of auto parts and contributes 5,6 percent to world
production equivalent to $\$ 82$ billion annually in production and $\$ 65$ billion in exports. The
state of Nuevo León contributes 20 percent of the country's manufacturing GDP in this
sector. Industry 4.0 and its pillars based mainly on technology, automation and the
internet of things presents a challenge to the country due to the insufficient investment
in the level two supplier companies (Tier 2) of the sector. The objective of this research
is to analyze the current and future status of Tier 2 companies belonging to the
Automotive Cluster of Nuevo León, Mexico in its process of incorporation into

Industria 4.0 en el sector de autopartes 
technological advances. A mixed technique was used to obtain data. In addition to the review of literature, semi-structured interviews with experts and as approach to the study, questionnaires to companies in the sector were conducted. With the above, is intended to answer the research question ¿Which actions should be considered to facilitate the incorporation of Industry 4.0 into auto parts in Nuevo Leon? It was found the slow incorporation of Tier 2 companies to technological advances and the lack of knowledge about their position in the industry. Some of the actions or strategies to facilitate this transition to the automation of manufacturing processes include partnerships between public and private sectors in alliance with the academic, as well as investment in research and development centers that allow the training and promotion of the employees on a permanent basis.

Key words: Auto parts, Industry 4.0, Tier 2, CLAUT, Nuevo Leon.

JEL Classification: L62, 031

Resumen: México es el quinto país productor de autopartes, y aporta 5,6 por ciento a la producción mundial equivalentes a 82'000 millones de dólares anuales en producción y $65^{\prime} 000$ millones en exportaciones. El estado de Nuevo León aporta el $20 \%$ del Producto Interno Bruto (PIB) manufacturero del país en este sector. La Industria 4.0 y sus pilares basados principalmente en tecnología, automatización y el internet de las cosas presenta un desafío debido a la insuficiente inversión en las empresas proveedoras nivel dos (Tier 2) del sector. El objetivo de esta investigación es analizar el estado actual y futuro de las empresas Tier 2 pertenecientes al Clúster Automotriz de Nuevo León en su proceso de incorporación a los avances tecnológicos. Se utilizó una técnica mixta para obtención de datos. Además de la revisión de literatura, se realizaron

Nava, K., Silva, A., Guajardo, A., Leyva U., \& Torres, C. 
entrevistas semiestructuradas a expertos y como aproximación al estudio, se aplicaron cuestionarios a empresas del sector. Con lo anterior, se pretende responder la pregunta de investigación ¿Qué medidas deben considerarse para facilitar la incorporación de la 14.0 en la industria de autopartes en Nuevo León? Entre los hallazgos se encontró la lenta incorporación de empresas Tier 2 a los avances tecnológicos y el desconocimiento de su nivel tecnológico en la industria. Entre las medidas por facilitar esta transición a la automatización de procesos de manufactura destaca las alianzas público privadas en acompañamiento con la academia, así como inversión en centro de investigación y desarrollo que permitan la capacitación y promoción de los empleados de forma permanente.

Palabras clave: Autopartes, Industria 4.0, Tier 2, CLAUT, Nuevo León.

\section{Introducción}

La industria automotriz en México está clasificada en cuatro categorías: vehículos ligeros; autopartes; autobuses, camiones y tracto camiones; y distribuidores. Cada una de estas categorías cuenta con asociaciones que impulsan el crecimiento de las empresas colaboradoras, tales como la Asociación Mexicana de la Industria Automotriz (AMIA), la Industria Nacional de Autopartes (INA), Asociación Nacional de Productores de Autobuses, Camiones y Tracto Camiones (ANPACT) y la Asociación Mexicana de Distribuidores de Automotores A.C (AMDA) (Oxford Business Group OBG, 2018; ProMéxico, 2015).

Industria 4.0 en el sector de autopartes 
Durante el año 2017, se produjeron 4,09 millones de vehículos automotores que representaron un incremento del $13 \%$ respecto al año anterior, siendo el principal sector receptor de inversión extranjera directa con $60^{\prime} 677$ millones de dólares y de mayor crecimiento de producción valorada aproximadamente de 90 millones de dólares, generando así, alrededor de 800'000 empleos (Clúster Automotriz CLAUT, 2018). Posteriormente, para el año 2018, de cada 100 vehículos producidos mundialmente, 4,2 fueron ensamblados en México.

Dado el crecimiento de sector automotor del país, muchas empresas extranjeras optan por trasladar sus plantas de producción a México por dos razones principales, la ventaja geográfica al ubicarse dentro del corredor de manufactura de Norteamérica que agiliza los procesos y los costos logísticos, y como segunda razón, los bajos costos en mano de obra.

En los últimos años, México se ha convertido en un gran polo de desarrollo para el sector automotriz y de autopartes a nivel global manteniéndose en un lugar atractivo para inversión de grandes compañías como General Motors, Fiat Chrysler México, Nissan Mexicana, Kia y Volkswagen de México convirtiéndose en un pilar importante para la economía del país fortaleciendo las capacidades de la industria local, desarrollo de infraestructura y la disponibilidad del capital

Nava, K., Silva, A., Guajardo, A., Leyva U., \& Torres, C. 
humano. Por lo anterior, el desarrollo de la industria automotriz debe ser prioridad y especialmente las habilidades deben ser ajustadas a las tendencias de producción de la Industria 4.0 (I 4.0), las cuáles son la digitalización, integración y personalización de sistemas y procesos.

Uno de los sectores más importantes de la industria automotriz es la industria de autopartes. El sector autopartes se clasifica en las Original Equipment Manufacturers (OEM), proveedores de primer nivel (Tier 1), proveedores de segundo nivel (Tier 2) y proveedores de tercer nivel (Tier 3 ). El sector de autopartes es de gran importancia, ya que el valor de sus exportaciones supera al valor del sector automotriz y tracto camiones juntos. Dentro del país existen diferentes regiones donde son fabricadas las autopartes. La región geográfica del bajío (constituida por los estados de Aguascalientes, Jalisco, Guanajuato, Querétaro y San Luis Potosí) ocupa el primer lugar en producción de autopartes con un 29,8 por ciento de la producción nacional. Mientras que a nivel estado, Coahuila ocupa el primer lugar a nivel nacional que produce el 18,9 por ciento del total fabricado en México, seguido por Chihuahua con un 13,9 por ciento y en tercer lugar Nuevo León con 8,3 por ciento (OBG, 2018).

Con respecto a la industria de autopartes en Nuevo León, estado fronterizo e industrial por excelencia, la Secretaría de Economía en el Centro de Estudios de las Finanzas Públicas (CEFP, 2017) del Gobierno Mexicano señala que el Clúster automotriz neoleonés es el más 
importante del país, debido a que en el 2016, el 9,2 por ciento de lo producido dentro de la industria automotriz en Nuevo León lo constituyeron partes para vehículos automotores como climas, sistemas automotrices, partes plásticas, partes para el sistema eléctrico y partes para motor y maquinados. Un año más tarde, Nuevo León produjo el 8,3 por ciento de autopartes en el país, siendo el quinto estado con más participación en la República Mexicana. Es importante resaltar que a pesar de no ser el estado con mayor producción este aporta el $20 \%$ del PIB manufacturero del país.

Por otra parte, la inclusión de la I 4.0, definida como el uso de altas tecnologías para la mejora de procesos de una empresa, es un paso importante para la evolución de la industria de autopartes, ya que adopta procesos de manufactura avanzada y cuenta con potencial para lograr una ágil adaptación de tecnologías en sus procesos, logrando así conservar su posición en diversas industrias. La I 4.0 permitirá facilitar el trabajo y buscará automatizar los procesos repetitivos y limitar los errores en las empresas manufactureras (ProMéxico, 2017).

A pesar de lo anterior, el rezago tecnológico en México es claro, ya que prácticamente no hay innovación tecnológica dentro de las plantas ensambladoras de autos o ésta es mínima. En promedio, las empresas

Nava, K., Silva, A., Guajardo, A., Leyva U., \& Torres, C. 
Tier 1, invierten hasta $5 \%$ de sus recursos en innovación, pero las proveedoras Tier 2 y las Tier 3, invierten menos.

Actualmente existe un déficit de mano de obra y talento especializado en Nuevo León, lo que afecta el desarrollo y vinculación con la I 4.0, ya que en menos de cinco años podría verse un cambio sustancial en la forma de trabajar, por lo que se debe de comenzar a capacitar a los trabajadores para ser parte de ésta nueva industria (García, 2018).

Por lo tanto, la problemática que se detecta en esta investigación es la insuficiencia tecnológica en las empresas manufactureras de autopartes proveedoras de segundo nivel en Nuevo León. A partir de esta problemática, se plantea como objetivo general, analizar el estado actual y el impacto de los avances tecnológicos en las empresas Tier 2 en procesos manufactureros de autopartes en Nuevo León relacionados con los nueve pilares de la I 4.0. Lo anterior, permitirá responder la siguiente pregunta de investigación: ¿Qué medidas deben considerarse para facilitar la incorporación de la 14.0 en la industria de autopartes en México? 


\section{Marco teórico}

\section{Industria Automotriz}

Desde 1965, año en el que fue firmado el acuerdo Canada-United States Automotive Products Agreement (AUTOPACT), se eliminaron los aranceles en productos automotrices, lo que implicó a las empresas transnacionales replantear la distribución de su producción, modificando la localización de producción y generando estrategias para dar uso a las ventajas competitivas de cada país. El cambio generado por este acuerdo fue el insertar un patrón geoeconómico, una división binacional de trabajo con la cual se establecieron economías de escala, grandes cantidades de producción para el abastecimiento del mercado canadiense y estadounidense, reduciendo los costos de producción (Klier \& Rubenstein, 2016).

Más tarde, durante la Crisis de 1982, una recesión causada por el aumento de las tasas de interés en Estados Unidos, ocasionó el estancamiento de la productividad estadounidense y competencia japonesa, obligando a reestructurar la industria automotriz de las 3-D (Ford, General Motors y Chrysler), el cierre de plantas y rescate de Chrysler por parte del gobierno de EU y Canadá. También, dio inicio a inversiones directas japonesas en Estados Unidos, lo cual generó como

Nava, K., Silva, A., Guajardo, A., Leyva U., \& Torres, C. 
resultado las negociaciones de G-7 en 1985. Dicha negociación obligó a Japón a la sobrevaluación de su moneda. Con yen fuerte y sorteando restricciones cuantitativas a la importación, las transnacionales automotrices japonesas eligieron establecer filiales para la producción de autos dentro del mercado norteamericano. Así llegaron las ensambladoras de vehículos a Estados Unidos como: Nissan, Toyota, Honda, Mitsubishi y Mazda; y en Ontario, Toyota, Honda y Suzuki (Dávalos, 2017).

Años más tarde, con la entrada en vigor el Tratado de Libre Comercio de América del Norte (TLCAN) en 1994, para Canadá fue un aspecto importante, qué gracias a la integración de México en la división regional de la industria, cuenta con salarios bajos como factor de atracción para ensambladoras de autos y con experiencia laboral en el ensamblaje. Gracias a este tratado comercial, se incrementó la producción de vehículos y dio un auge continental en la industria de los tres países.

Las principales razones por las que se impulsó el TLCAN fueron el poco crecimiento en la producción de ciertas manufactureras en los Estados Unidos y la posibilidad de la transferencia de la tecnología a otras regiones que contaran con mano de obra más barata. Asimismo, los países asiáticos estaban retomando fuerza y era necesario crear una 
economía de manufactura competitiva para hacerle frente a los nuevos competidores (Navarrete-Báez, 2016).

Con la llegada del nuevo milenio y la entrada en vigor del Tratado de Libre Comercio con la Unión Europea, la industria automotriz mexicana se posicionó como la más importante en América Latina, triplicando las exportaciones de vehículos ligeros en comparación a lo destinado el mercado nacional. Además, en América Latina dio un gran impulso a esta industria gracias a la entrada en vigor del Mercado Común del Sur (MERCOSUR), un proceso de integración regional fundado por Argentina, Brasil, Paraguay y Uruguay, muchas empresas armadoras invirtieron en la expansión de sus plantas en los países latinoamericanos, ya que se implementaron y modernizaron procesos en la cadena de suministro que facilitó y agilizó la producción de vehículos ligeros en la zona.

Por otro lado, para el año 2003 durante la administración del entonces Presidente de México, Vicente Fox Quezada consciente de la importante apertura comercial propiciada por los diferentes acuerdos comerciales firmados, se publica el "Decreto para el apoyo de la competitividad de la industria automotriz terminal y el impulso al desarrollo del mercado interno de automóviles", en el cual se reconoció que México en ese momento estaba preparado para satisfacer la

Nava, K., Silva, A., Guajardo, A., Leyva U., \& Torres, C. 
demanda de vehículos y la necesidad de crear mecanismos que propiciaran el incremento de la competitividad del sector buscando el fortalecimiento interno del mercado antes de pensar el mercado global. Con esto, se plantearon objetivos para impulsar aún más el desarrollo de la industria, por ejemplo, instruir la disminución de costos de las importaciones, además se autorizó a las empresas a importar mayores cantidades de materia prima con un arancel preferencial, siempre y cuando estas empresas se comprometieron a aumentar la inversión en el país.

Más tarde, en el sexenio del presidente de México (2006-2012) Felipe Calderón, se enfocó en mantener y posicionar a la industria automotriz como una de las más importantes a nivel mundial. No fue fácil de conseguirlo ya que para el 2008 se desencadenó una de las peores crisis financieras, lo que causó un impacto negativo en la economía mundial y más en la mexicana, provocando que el sector automotriz descendiera a la novena posición de los productores más importantes de automóviles a nivel mundial. Asimismo, no se registraron importantes inversiones extranjeras directas, debido a la ola de inseguridad que azotaba al país en ese momento. Muchos inversionistas no se animaban a entrar al país puesto que no querían poner en riesgo la integridad de sus trabajadores, fue hasta el año de 2011 en el cual las principales armadoras instaladas en el país (Ford, Nissan y Volkswagen) invirtieron

Industria 4.0 en el sector de autopartes 
en la expansión y modernización de sus plantas, para poder satisfacer la demanda de vehículos a nivel mundial. Para el 2014, México ya se posicionaba como el séptimo productor de autopartes a nivel internacional, y el número uno en América Latina, sobrepasando a Brasil (ProMéxico, 2016). En 2016, Kia Motors y Audi anunciaron el inicio de operaciones en el país, las que, en conjunto aportaron 450'000 unidades adicionales a la producción del país (Duran et al., 2018).

Durante la renegociación del TLCAN que inició en 2017 y continuó durante el año 2018, se generó una versión actualizada del tratado llamada T-MEC (Tratado entre México, Estados Unidos y Canadá), firmado por los tres países y en espera de la autorización de sus congresos respectivos en el 2019. El T-MEC estableció para el sector automotriz un incremento de valor de contenido regional de las autopartes producidas en la región en $75 \%$ en comparación con el $62,5 \%$ que se manejaba con el tratado anterior. De igual manera, para el 2023 se estableció que el $40 \%$ del valor del vehículo tendría que ser fabricado en áreas donde los salarios fueran de 16 dólares por hora, situación que es cubierta por Estados Unidos y Canadá pero no por México y en donde los salarios más bajos en las plantas pueden llegar a ser de 8-9 dólares la hora. El valor de contenido laboral (VCL), será de $40 \%$ para autos y de $45 \%$ para camionetas. Otro punto importante es que los fabricantes

Nava, K., Silva, A., Guajardo, A., Leyva U., \& Torres, C. 
deberán obtener al menos el $70 \%$ de su aluminio y acero de los tres países participantes (Bergsten, 2017; Gobierno de México, 2018; OBG, 2018).

Por otra parte, durante el 2018 en el Acuerdo Amplio y Progresista de Asociación Transpacífico (CPTPP), 11 países alcanzaron un acuerdo. Este acuerdo nace del Acuerdo de Asociación Transpacífico (TPP), que tiene por objeto la creación de una plataforma para una integración económica con la región Asia-Pacífico. Este tratado será un catalizador para la industria nacional, en especial para la de autopartes (Amador, 2018).

Por otro lado, según la Secretaría de Economía a través de ProMéxico (2016), las perspectivas de la industria automotriz a nivel global y la forma en cómo tienen que ser consideradas por las industrias mexicanas son enumeradas de la siguiente manera:

1. Tecnología de motores y la transición a lo eléctrico.

2. El cambio de lo mecánico a lo electrónico. Mayor grado de integración de los OEMs o armadoras con la industria electrónica (componentes).

3. Se espera que hacia 2020, los vehículos híbridos tendrán mayor participación que los eléctricos, pero las tendencias indican un mundo completamente eléctrico en el largo plazo. 
La industria automotriz ha logrado posicionarse mundialmente como uno de los principales fabricantes de vehículos ligeros, convirtiéndose en el séptimo mayor productor y cuarto mayor exportador, que en el 2017 contribuyó con el 2,9 por ciento del PIB nacional y 18,3 por ciento del manufacturero, generando impactos en 157 actividades económicas del país, 84 correspondientes a la industria manufacturera y 73 a comercio y servicios. De igual manera, el PIB automotriz creció 4,6 veces más que el nacional $(9,4$ por ciento contra 2,0 por ciento, en comparación con el año anterior (AMIA, 2018).

Asimismo, la industria de autopartes en México conserva una tendencia de crecimiento sostenido en los últimos cinco años debido que el sector ha alcanzado cifras récord en producción de $82^{\prime} 000$ millones de dólares anuales y $65^{\prime} 000$ millones de dólares anuales por concepto de exportaciones. México se ha convertido en el quinto país productor de autopartes en el mundo con $75 \%$ de piezas manufacturadas enviadas a Canadá y principalmente a Estados Unidos, contando con un gran impacto a nivel mundial, exportando el $67 \%$ de su producción, equivalente al $5,6 \%$ de la producción mundial (OBG, 2018).

En los últimos años, México se convirtió en un gran polo de desarrollo para el sector automotriz y continúa manteniéndose como un lugar atractivo para inversión de grandes compañías de este sector y de

Nava, K., Silva, A., Guajardo, A., Leyva U., \& Torres, C. 
autopartes a nivel global (Barrera y Pulido, 2016; Vargas, 2016). Las empresas de autopartes del país en la industria terminal de vehículos ligeros tienen un total de 20 complejos de manufactura en 14 estados, con actividades desde ensamble y blindaje, fundición y el estampado. La industria acepta nuevos desafíos al enfocar esfuerzos en el desarrollo de proveedores tier 2 y tier 3 de la cadena. La tendencia se denota en los caminos de alta especialización de procesos de manufactura y componentes para asegurar más valor agregado que contengan actividades de investigación, diseño y desarrollo. No sólo es esencial impulsar la disponibilidad de capital humano capacitado sino capacidades de innovación, diseño y desarrollo de una industria como lo es la industria automotriz.

Sin embargo, México no cuenta con suficiente capacidad interna para el abastecimiento de armadoras, ya que sólo cuenta con 400 tier 2 y 400 tier 3, y están por debajo de las 1200 que deberían del existir de cada una para llegar a satisfacer la demanda del presente y futuro (OBG, 2018). Asimismo, se señala que es importante el desarrollo de proveedores tier 1 y tier 2 para poder integrar a las pequeñas y medianas empresas a cadenas productivas de grandes empresas tractoras en el Estado de Nuevo León. Es importante mencionar, que las empresas tractoras son las que impulsan el crecimiento y desarrollo económico a un nivel macro, la mayoría de estás son nacionales o internacionales. 
Desde el sector gobierno, la Secretaría de Desarrollo Económico de Nuevo León tiene como misión ampliar el programa de capacitación para las tier 2 y así poder convertir al Estado en uno de los principales motores de la industria manufacturera en la república mexicana (Nava, 2014).

Figura 1. Línea del tiempo de la industria automotriz mexicana

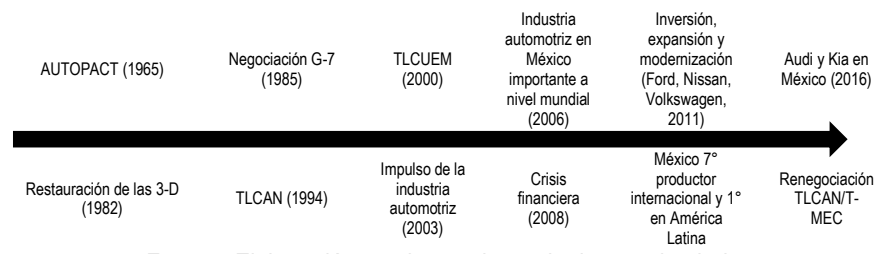

Fuente: Elaboración propia con datos citados en el trabajo.

Industria 4.0

La Cuarta Revolución Industrial comúnmente relacionada con la Industria 4.0 (I 4.0) tiene sus orígenes en Alemania, específicamente en la feria de la tecnología "Hannover Messe" que inició en 1947. El físico alemán Henning Kagermann fue uno de los principales promotores del concepto Industria 4.0 que busca promueve la digitalización y el internet de las cosas en la producción industrial. Nació como una propuesta de economía política basada en estrategias fundamentadas para el uso de

Nava, K., Silva, A., Guajardo, A., Leyva U., \& Torres, C. 
alta tecnología que busca la unificación de tecnologías de información para mejorar procesos (ProMéxico, 2017). Posteriormente, se creó el Industry 4.0 Maturity Index que es un índice que provee lineamientos a las empresas para que puedan determinar su nivel de adopción a esta industria. Este concepto conformó lo que hoy en día es conocida como la Industria 4.0 (I 4.0) que busca la comunicación vía internet, permitiendo la interacción e intercambio de información entre personas, personas y máquinas e incluso máquinas y máquinas (Roblek, Mesko, y Krapez, 2016). La I 4.0 deberá incorporar la digitalización de productos, automatización en los procesos, así como la interconexión de los sistemas de manufactura a las cadenas de suministro de las empresas (Basco, et al., 2018).

La digitalización permitirá que la | 4.0 transforme la manera de operar los procesos y operaciones. Incorpora nueve tecnologías que permitirán la interconexión entre sensores, maquinas, infraestructura y sistemas. Gerbert, Lorenz, Rußmann y Waldner (2015) mencionan que los nueve pilares de la I 4.0 son los robots autónomos, simulaciones, sistemas de integración vertical y horizontal, el internet industrial de las cosas, ciberseguridad, computación en la nube, manufactura aditiva, realidad virtual y análisis de Big Data. Todos estos pueden ser entendidos como el conjunto de herramientas que permitirán impulsar la 
transformación de la industria y que se presentan como los pilares sobre los cuáles se construirá este nuevo escenario (Castresana, 2016).

Es importante mencionar que la | 4.0 nació de dos pilares principalmente, el internet de las cosas y sistemas de integración vertical y horizontal, también conocidos como sistemas ciber físicos (Roblek, Mesko y Krapez, 2016). El primer concepto permite conectar a internet cualquier objeto y por otro lado, el siguiente permite la conexión de objetos entre el mundo real y el físico. Estos dos fenómenos funcionan en conjunto ya que el primero permite la conexión entre máquinas y personas, mientras que el segundo brinda una plataforma virtual en donde se pueden realizar estas funciones. Esto quiere decir que las tecnologías que involucran objetos tales como la realidad aumentada, simulaciones, robots autónomos y fabricaciones aditivas deben de hacer uso de estas plataformas para realizar sus funciones.

La realidad aumentada es una de las aplicaciones tecnológicas más utilizadas en el ámbito industrial, la cual facilita la elaboración de tareas y sirve como un manual digital e interactivo. Un claro ejemplo de esta tecnología son las gafas inteligentes. En el ámbito de la industria automotriz, estás pueden ser utilizadas para identificar y corregir errores en la fabricación de un motor, chasis y transmisión entre otras piezas (Becerril, 2018). Un concepto parecido al anterior es la simulación, una

Nava, K., Silva, A., Guajardo, A., Leyva U., \& Torres, C. 
tecnología que permite trasladar el mundo real al mundo virtual y posibilita la visualización virtual de máquinas, piezas y hasta líneas de producción.

Por otro lado, existe la robótica como una alternativa para facilitar trabajos complejos. Aunque en un principio la finalidad de los robots era trabajar por sí solo en tareas específicas, la robótica está tomando un giro colaborativo con la finalidad de hacer posible la combinación de las capacidades de un robot con la inteligencia y habilidades de una persona (Gerbert et al., 2015). Este pilar ofrece flexibilidad a la hora de automatizar las fábricas, ya que un robot puede reconfigurarse y reutilizarse para diferentes operaciones. Además, estos robots servirán como una fuente de obtención constante de datos, lo que mejorará la toma de decisiones.

Otro de los pilares más conocidos y utilizados actualmente en la industria es la fabricación aditiva. Castresana (2016) menciona que los sectores que más utilizan esta tecnología son la industria y los bienes de consumo, seguido por la medicina. De igual manera, menciona que la fabricación aditiva consiste en la edición sucesiva de material, normalmente en polvo, en capas muy finas una sobre otra hasta formar un producto sólido. Un ejemplo de esto es la impresión 3D, la cual es utilizada en la industria para la impresión de materia prima o diseños complejos de piezas pequeñas. Cabe mencionar que uno de los principales atributos de la fabricación aditiva reduce los costos de 
transportación, la cadena de suministro y permite un mayor control de inventarios (Gerbert et al., 2015).

El uso de estas nuevas tecnologías traerá consigo una gran cantidad de manejo de datos en las plataformas digitales y herramientas como el Big Data permitiendo a las empresas identificar patrones y análisis de todos los datos recolectados en los espacios ciber físicos. En otras palabras su análisis permitirá identificar patrones e interdependencias, analizar los procesos y descubrir ineficiencias e incluso predecir eventos futuros (Val, 2016). El Big Data buscará cumplir y desarrollar uno de los principales objetivos de la I 4.0 como es el servicio al cliente.

Por otro lado, la computación en la nube permite el acceso a una red compartida de recursos de computación, la cual puede ser utilizada con poco esfuerzo e interacción mínima con el proveedor de servicio. Aunque las empresas ya utilizan software que operan en la nube, con la I 4.0 habrá más necesidad de compartir información entre sitios y quizá hasta entre compañías. Estas tecnologías en la nube mejorarán y tratarán de enfocarse más hacia la producción. Algunas de las ventajas de la tecnología en la nube son la incorporación de la información, reducir los gastos en hardware y software, mayor flexibilidad en recursos

Nava, K., Silva, A., Guajardo, A., Leyva U., \& Torres, C. 
tecnológicos, aumento de la capacidad informática, diversificación de los sistemas, entre otros (Castresana, 2015; Zegarra y Perez, 2018).

Uno de los mayores desafíos de la l 4.0 es la protección de datos. Un mayor uso de tecnologías y manejo de información virtual representa un riesgo debido a los conocidos "hackers". Existe una preocupación en la forma en que las empresas van a garantizar la privacidad y seguridad de los datos de otras empresas (Roblek, Mesko y Krapez, 2016). Así mismo, se debe contar con una ética profesional muy alta para hacer negocios en un futuro.

Clúster Automotriz de Nuevo León (CLAUT)

Una estrategia para promover el crecimiento de la industria automotriz en el estado de Nuevo León, México es el clúster automotriz. Un clúster es definido como una concentración geográfica de empresas e instituciones de un campo en particular, vinculadas por características comunes, que compiten, pero a su vez cooperan entre sí. Existen diferentes clústeres alrededor de la república mexicana, los cuales están clasificados de acuerdo a las categorías pertenecientes de la Industria Automotriz, las cuales son vehículos ligeros y sus motores; vehículos pesados y sus motores; y centros de investigación y desarrollo (Unger y Chico, 2002). 
Tabla 1. Clústers en México

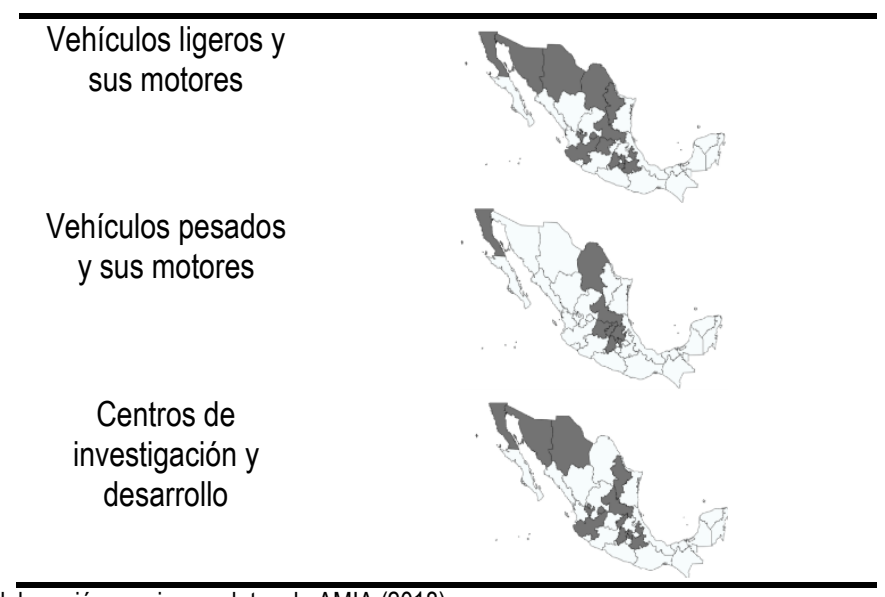

Fuente: Elaboración propia con datos de AMIA (2018).

El Clúster Automotriz de Nuevo León (CLAUT) nació en 2007, y es una asociación civil que se compone por fabricantes de primer nivel de la industria automotriz, instituciones académicas y gubernamentales relacionadas con el ramo. El CLAUT tiene como misión el impulsar la competitividad del sector en la región a través de la colaboración y vinculación entre gobierno, academia y empresa, buscando un crecimiento sostenible e impulsado en desarrollo de México.

Nava, K., Silva, A., Guajardo, A., Leyva U., \& Torres, C. 
Entre los miembros del CLAUT se distinguen 5 Original Equipment Manufacturers (OEMs), 29 proveedores de primer nivel (tier 1), 56 proveedores de segundo nivel (Tier 2), 8 universidades, 2 centros de investigación y 3 instituciones de gobierno.

Actualmente en el estado de Nuevo León están instaladas 66 empresas proveedoras de primer nivel (T1), de las cuales 29 están afiliadas al CLAUT. Mientras que proveedoras de segundo nivel (T2) existen 188 de las cuales 56 están afiliadas al CLAUT.

De igual manera el CLAUT trabaja por medio de comités, cada uno comprendido por un grupo de representantes de empresas, instituciones y gobierno, que desarrollan temas de interés, generando proyectos de valor para impulsar el sector automotriz en la región. Actualmente operan 21 grupos de trabajo, organizados en 8 comités y 13 subcomités. Algunos de los temas de interés son: la cadena de suministro, innovación, desarrollo de proveedores y Tier 2, entre otros (CLAUT, 2018).

\section{Metodología}

La investigación es de tipo mixto. En una primera etapa, se utilizó el método cualitativo mediante dos instrumentos de recolección de datos. En primer lugar, se llevó a cabo una revisión de literatura que consistió en la lectura y análisis de textos, reportes y publicaciones. Como segundo 
instrumento, se recopilaron datos de fuentes primarias mediante el diseño y realización de entrevistas semiestructuradas. Se realizaron a conveniencia cuatro entrevistas a representantes clave del sector: Luis Ochoa representante Comité de Tier 2 del CLAUT, Rodrigo Martinazioli, Coordinador del Comité de la Cadena de Suministro, Empaque y Comercio Exterior del CLAUT, Abraham Tijerina, Coordinador de la Iniciativa Ciudadana de la Industria 4.0 del Gobierno de Nuevo León, y Andrés Hernández, profesor-investigador de la Universidad de Monterrey con especialización en robótica y ciberseguridad. "En ciertos estudios es necesaria la opinión de expertos en un tema. Estas muestras son frecuentes en estudios cualitativos y exploratorios para generar hipótesis más precisas o la materia del diseño de cuestionarios" (Hernández, Fernández y Baptista, 2014, p. 387). La información obtenida se procesó a través de un análisis de contenido.

En una segunda etapa y como una primera aproximación a un análisis futuro del universo de las Tier 2 pertenecientes al CLAUT en Nuevo León, se aplicó un cuestionario en línea. Para esta investigación se tomó como muestra la respuesta de solo ocho empresas. Las empresas pertenecen al sector de autopartes catalogadas como proveedoras segundo nivel de las armadoras. De la muestra, cuatro empresas son medianas (51-250 empleados), tres son grandes (251 o

Nava, K., Silva, A., Guajardo, A., Leyva U., \& Torres, C. 
más empleados) y solamente una de ellas es microempresa (1-10 empleados), todas ellas con inversión $100 \%$ mexicana. El acercamiento a estas empresas se logró gracias al apoyo del CLAUT quien a través de sus bases de datos se logró enviar el cuestionario a sus socios.

Para procesar la información se utilizó estadística descriptiva que permitió conocer la relación y frecuencia de los nueve pillares de la 14.0 en el sector de autopartes de Nuevo León. Se elaboraron tablas para una representación más grafica de los resultados. Posteriormente se analizaron los hallazgos desde las perspectivas de gobierno, academia e industria. Aunque el cuestionario no pretende generalizar los resultados, si presenta una aproximación de las empresas del sector que seguirán estudiándose en una investigación posterior.

\section{Resultados}

Los hallazgos se definieron desde tres distintos sectores en la industria de autopartes gobierno, academia e industria. En las diferentes perspectivas de análisis existe consenso en afirmar que existe una relación entre la industria de autopartes con la Industria 4.0, pero antes de pensar en introducir estas nuevas tecnologías, las empresas deben de enfocarse en los conceptos más básicos como lo son el almacenamiento y la automatización. Incluso existe una falta de colaboración entre las 
personas que desarrollan los sistemas tecnológicos y las pertenecientes a la industria (tier 2) lo cual los limita para poder implementar los nuevos sistemas y avances tecnológicos.

No obstante, el sector industrial y académico coinciden que la mayoría de las empresas desconocen la cantidad de tecnologías que se pueden implementar en sus procesos, especialmente aquellas relacionadas a la 14.0. Los representantes tanto el gobierno como la industria mencionan que como consecuencia de la falta de información y conocimiento, las empresas Tier 2 no se han alineado con la I 4.0, ya que es necesario, "cambiar la forma de pensar y la manera de hacer las cosas debido que es indispensable no solo introducir nuevas tecnologías, si no generarlas" (Entrevista Rodrigo Martinazioli, 2019).

Desde la óptica de gobierno, aunque las empresas Tier 2 están interconectadas, carecen de un sistema inteligente ya que no cuentan con lo más básico, como el conectar el ERP con el sistema de producción y/o la demanda afectando su competitividad. Además de la limitante por falta de información y conocimiento, se suma el financiamiento para dichos propósitos de la industria. Tanto el sector gobierno y la industria indican que la limitación secundaria es el talento del capital humano, ya que no se cuenta con la tecnología necesaria para desarrollarla al máximo. El capital humano se verá desplazado por las nuevas tecnologías de la 14.0,

Nava, K., Silva, A., Guajardo, A., Leyva U., \& Torres, C. 
pero estos mismos se reubicarán dentro del sistema organizacional, permitiéndoles desarrollar nuevas habilidades.

Con el fin de apoyar a las empresas que buscan introducir estas nuevas tecnologías a sus procesos, el Clúster Automotriz y el gobierno (Programa Iniciativa Nuevo León 4.0) apoyan a las empresas a través de cursos, capacitaciones e información e incluso, el programa ofrece un apoyo económico a las empresas participantes. En estos eventos gubernamentales se ven temas como Big Data, el internet de las cosas, inteligencia artificial, simulaciones, robótica, manufactura aditiva y ciberseguridad. De igual manera, el gobierno cuenta con un centro de capacitación para los trabajadores, mejor conocido como el Centro de Innovación, Soluciones Empresariales y Tecnológicas (CISET), el cual ofrece programas para certificar empresas y dar apoyos a crédito con garantía, beneficios que muchas empresas no conocen. A pesar de todas estas facilidades, tanto el sector industria como gobierno reconocen la falta de interés por parte de las Tier 2 al querer sumarse a esta nueva transformación ya que temen saber su posición actual tecnológicamente hablando y su capacidad de inversión es limitada.

De igual manera, tanto el gobierno, la academia y el sector privado coinciden que la nueva transformación es una decisión que no se debe de pensar, simplemente sumarse si las empresas Tier 2 pretenden continuar dentro del sector. Por tanto, si se tiene una empresa afiliada a 
un clúster es indispensable aprovecharlo al máximo, de lo contrario es necesario integrarse a alguno para que en conjunto se logre un mayor aprovechamiento tecnológico. En otras palabras, las redes de contacto y alianzas facilitan el conocimiento e implementación de tecnologías avanzadas en los procesos y operaciones de las industrias.

Ahora bien, el acercamiento que se tuvo con las empresas Tier 2 permitió identificar el estado que guardan los pilares de la Industria 4.0. Dentro de los principales hallazgos, se obtuvo que el pilar más utilizado dentro de las empresas es la computación en la nube. Asimismo, el internet de las cosas y las simulaciones son utilizados solo en tres de las ocho empresas que participaron en el cuestionario.

Gráfica 1. Tecnologías utilizadas en empresas Tier 2

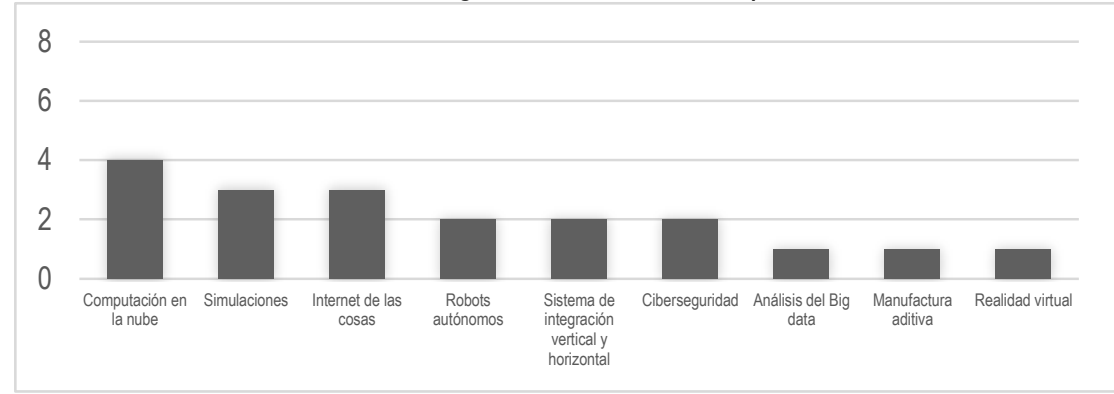

Fuente: Elaboración propia

Nava, K., Silva, A., Guajardo, A., Leyva U., \& Torres, C. 
De igual manera, cada una de las empresas cuenta con tecnologías utilizadas en distintos departamentos. La tabla 2 muestra cuántas empresas utilizan un pilar en específico en cada uno de los departamentos analizados, los cuales son producción, calidad, recursos humanos, finanzas, compras, logística externa, logística interna y planeación.

Tabla 1. Tecnologías utilizadas en empresas Tier 2 por departamento

\begin{tabular}{|c|c|c|c|c|c|c|c|c|}
\hline & $\begin{array}{l}\text { Producció } \\
n\end{array}$ & $\begin{array}{c}\text { Calida } \\
d\end{array}$ & $\begin{array}{c}\text { Recursos } \\
\text { Humano } \\
s\end{array}$ & $\begin{array}{c}\text { Finanza } \\
s\end{array}$ & $\begin{array}{c}\text { Compra } \\
s\end{array}$ & $\begin{array}{c}\text { Logistic } \\
a \\
\text { Externa }\end{array}$ & $\begin{array}{l}\text { Logistic } \\
\text { a Interna }\end{array}$ & $\begin{array}{c}\text { Planeació } \\
n\end{array}$ \\
\hline 1. Análisis del Big Data & 3 & 2 & 2 & 2 & 1 & 2 & 2 & 2 \\
\hline 2. Robots autónomos & 2 & 1 & - & - & - & 1 & - & - \\
\hline $\begin{array}{l}\text { 3. Sistema de integración vertical y } \\
\text { horizontal }\end{array}$ & 2 & 2 & 2 & 1 & 1 & - & 1 & 1 \\
\hline 4. Ciberseguridad & 2 & 2 & 3 & 4 & 3 & 1 & 3 & 2 \\
\hline 5. Computación en la nube & 1 & 1 & 1 & 1 & 2 & 1 & 2 & 1 \\
\hline 6. Manufactura aditiva & 1 & - & - & - & 1 & 1 & - & - \\
\hline 7. Realidad virtual & 2 & - & - & - & - & - & - & 1 \\
\hline 8. Simulaciones & 2 & - & - & - & - & 1 & - & - \\
\hline 9. Internet de las cosas & 1 & . & . & - & . & - & - & . \\
\hline
\end{tabular}

Fuente: Elaboración propia.

Se puede observar que el pilar más utilizado es la ciberseguridad, específicamente en el departamento de finanzas. Esto demuestra que la 
mitad de las empresas tiene como prioridad la protección de datos en este departamento. El análisis del Big Data, la ciberseguridad y la computación en la nube son utilizadas por una o más empresas en todos los departamentos evaluados, esto quiere decir que hay una tendencia hacia el análisis, protección y almacenamiento de datos dentro de una empresa.

El departamento de producción es en el que por lo menos una empresa utiliza los pilares de la 14.0, siendo el análisis del Big Data la tecnología más utilizada. Tres de las ocho empresas utilizan esta tecnología en sus procesos de producción. En este mismo departamento se tiene que dos de las ocho empresas utilizan robots autónomos, sistemas de integración vertical y horizontal, ciberseguridad, realidad virtual y simulaciones.

Es importante mencionar que el pilar menos utilizado es el internet de las cosas, uno de los pilares principales de la I 4.0. De las ocho empresas, solamente una de ellas utiliza esta tecnología. Además, esta empresa solamente la utiliza en el departamento de producción. Por otro lado, los pilares de robots autónomos, manufactura aditiva, realidad virtual y simulaciones se utilizan en menos de la mitad de los departamentos evaluados.

Nava, K., Silva, A., Guajardo, A., Leyva U., \& Torres, C. 
Con lo que respecta a la incorporación de los pilares 14.0, tres de las ocho empresas consideran que estas tecnologías se están utilizando de manera efectiva y son suficientes para mejorar la eficiencia en sus procesos y costos. Sin embargo, una empresa considera que las tecnologías no son utilizadas de manera eficiente.

Cabe mencionar que para estas empresas, dos de los retos más significativos al introducir estas tecnologías, son la capacitación del personal y la implementación de las nuevas tecnologías. La mayoría considera que la mejora de estas tecnologías puede ser implementada en un corto o mediano plazo. Tres empresas consideran que la implementación puede tomar menos de un año mientras que cuatro de ellas consideran que esta implementación puede tomar de uno a tres años. Por otra parte, con lo que respecta al capital humano, todas las empresas consideran que sus empleados deben de contar con conocimiento de software y capacidad para analizar datos. De la misma forma, la mayoría de las empresas consideran que otro atributo importante es la organización, por encima de la programación en donde solamente cuatro empresas consideraron este atributo como necesario. En la gráfica 6 se puede observar lo anteriormente mencionado. 
Gráfica 2. Habilidades requeridas para trabajar con tecnologías 4.0

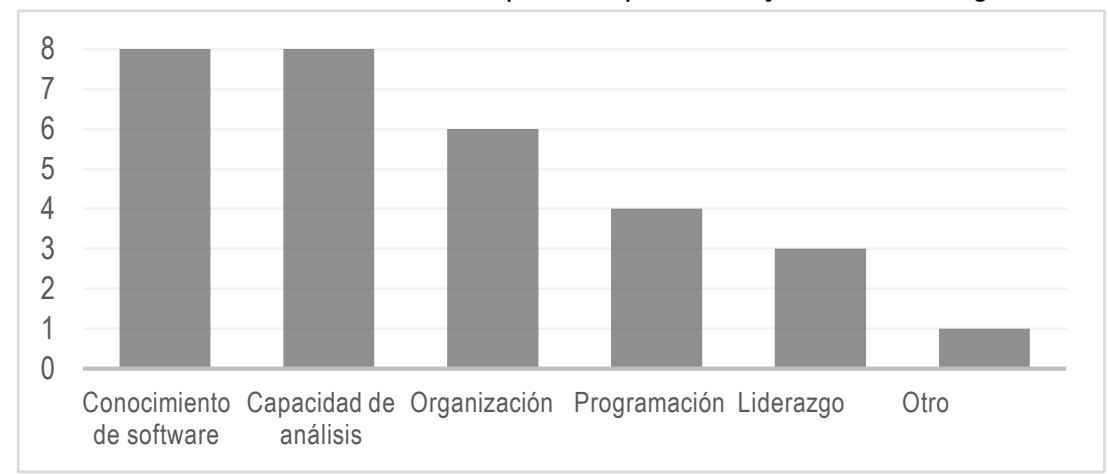

Fuente: Elaboración propia.

\section{Conclusión}

Es esencial que exista una conexión entre los sectores industria, gobierno y académico, es decir, una estrategia de vinculación permanente y no ocasional o por proyecto. Se identificó que las empresas no cuentan con el conocimiento del significado de la industria 4.0 aunque el gobierno brinde capacitaciones y programas para informar a las empresas acerca de estas nuevas tecnologías (Entrevista a Héctor Tijerina, 2019). Esto se relaciona con la respuesta de la industria, ya que como podemos observar

Nava, K., Silva, A., Guajardo, A., Leyva U., \& Torres, C. 
en la tabla 2, se hace muy poco uso de los pilares de la industria 4.0 en los departamentos analizados. Un claro ejemplo de esto es que el pilar más utilizado dentro de un departamento es la ciberseguridad, y esto solamente representa la mitad de las empresas.

Sin embargo, si las empresas Tier 2 no se adaptan a esta revolución, el principal riesgo será la pérdida de competitividad actual con base al mercado (Entrevista a Rodrigo Martinazioli, 2019). Sin embargo, el capital humano se podría ver afectado por la I 4.0, ya que existe un riesgo de desempleo, pero las empresas deben de buscar soluciones a este problema, como lo hizo la empresa Toyota, fundando un instituto especializado para sus trabajadores, donde se capacitan con diferentes programas, para finalmente reubicar a los empleados en diferentes áreas de la empresa. El instituto de investigación Toyota es ampliamente reconocido por su vínculo con la academia e investigación para enfrenar los desafíos del desarrollo del sector sobre todo tecnológico.

Por otro lado, en el sector académico ya se están trabajando y aplicando proyectos de automatización, robótica, ciberseguridad, entre otras tecnologías innovadoras. Una asociación entre los sectores de gobierno y académico beneficiaría a todos los sectores y mejoraría los procesos y operaciones de las empresas. Un ejemplo de lo anterior se observa en la empresa minera Rio Tinto en Australia, donde la empresa contrata alumnos durante sus estudios profesionales y de posgrado para

Industria 4.0 en el sector de autopartes 
la implementación de proyectos de mejora dentro de sus procesos como el de vehículos autónomos, con el beneficio de la obtención de becas del $100 \%$ para sus estudios. Asimismo, el país debe de crear este tipo de programas ya que los programas de licenciatura y maestría están comenzando a incluir en sus planes de estudios asignaturas relacionadas con la | 4.0, tales como programación, análisis de datos y automatización. Los egresados cuentan con las herramientas necesarias para innovar y aplicar cambios significativos dentro de las empresas y es algo que las empresas tienen que aprovechar (Entrevista a Andrés Hernández, 2019). Hoy en día, Nuevo León, ya cuenta con un avance en la academia incorporando temas clave para enfrentar las nuevas dinámicas de la manufactura y avance tecnológico, sin embargo, se necesita avanzar más rápido y crear más alianzas con otros institutos en el extranjero.

Por lo tanto, las empresas tendrán que promover la capacitación a sus trabajadores para estar calificados para la toma de decisiones en sus procesos de manera correcta. Las empresas Tier 2 aún se encuentran un paso atrás de la industria 4.0 ya que desconocen la posición que guardan en los pilares que integran la I 4.0 (Entrevista a Héctor Tijerina, 2019).

Por lo tanto, las empresas deben de empezar a tomar conciencia sobre la importancia de la implementación de estas nuevas tecnologías y

Nava, K., Silva, A., Guajardo, A., Leyva U., \& Torres, C. 
deben de informase sobre los beneficios que proveen a los distintos departamentos de la empresa. Las empresas que no se sumen a esta transformación digital podrían quedar rezagadas de manera importante ante aquellas que decidas sumarse, pues el uso de las tecnologías representa una reducción en costos y una mejora en tiempos de producción. De igual manera, las empresas deben de visualizar estas tecnologías como una oportunidad de crecimiento y mejora en procesos, no como un gasto sin recompensa.

Por otra parte, existe una tendencia a creer que el capital humano se podría ver afectado por la incorporación de la 14.0 al sector manufacturero ya que existe el riesgo de desempleo. Esto no necesariamente es un riesgo es una oportunidad que México deberá saber capitalizar. Otros países como Alemania y Japón han sabido aprovechar los desafíos del desarrollo en beneficio de sus trabajadores. Por ejemplo, la construcción de centros de investigación para impulsar la capacitación y promoción de sus trabajadores. Desde la perspectiva académica, las universidades ya hacen su parte y muestra de ellos son las iniciativas conjuntas que se tienen con la industria en proyectos nacionales e internacionales en automatización, robótica, ciberseguridad, entre otras tecnologías innovadoras.

En suma, una red más integrada entre los sectores de gobierno, industria y académico seguirá siendo el primer paso para beneficiar al 
país. Los jóvenes deberán integrarse a la industria como parte de su preparación académica. La capacitación de los jóvenes fuera del aula en México y el extranjero será el tema por consolidar no solo desde la perspectiva académica pero también el industrial.

Finalmente, es importante señalar que la investigación retomará el cuestionario aplicado con la intención de explorar a profundidad la perspectiva de las empresas Tier 2 restantes y pertenecientes al CLAUT y que no fueron incluidas en este estudio.

\section{Referencias}

Amador, O. (13 de abril de 2018). EI TPP catalizará la industria automotriz. Periódico EI Universal. Disponible en: https://www.eleconomista.com.mx/empresas/ElTPP-catalizara-la-industria-automotriz-20160413-0017.html

Asociación Mexicana de la Industria Automotriz AMIA (2018). Conociendo la Industria Automotriz. Recuperado en http://www.amia.com.mx/

Barrera, A. y Pulido, A. (2016). La industria automotriz mexicana: situación actual, retos y oportunidades. ProMéxico, (1), 30-40

Basco, A., Beliz, G., Coatz, D., y Garnero, P. (2018). Industria 4.0: Fabricando el Futuro. Buenos Aires: Banco Interamericano de Desarrollo

Becerril, D. (2018). Sector automotriz lidera la adopción de Industria 4.0. El Economista Bergsten, F. (2017). Trade Balances and the NAFTA Renegotiation. Peterson Institute for International Economics. Recuperado en https://piie.com/system/files/documents/pb17-23.pdf

Nava, K., Silva, A., Guajardo, A., Leyva U., \& Torres, C. 
Castresana, C. (2016). Industria 4.0. Universidad de la Rioja, (1), 4-11

Centro de Estudios de Finanzas Públicas (2017).La Industria Automotriz en México y el Tratado de Libre Comercio con América del Norte (TLCAN). Disponible en: http://www.cefp.gob.mx/publicaciones/boleco/2017/becefp0072017.pdf

Cluster Automotriz de Nuevo León A.C. (2018). Boletín Mensual. Disponible en: https://indd.adobe.com/view/cd88fdd4-26bb-4cc6-bdcd-a8b568a6097c

Dávalos, E. (2017). La industria automotriz en América del Norte después de la crisis 2008: efectos en Canadá. Ola Financiera, 10(26), 117-128

Durán, N., Murrieta, J., García, J., Obando, J. y Jarcia, M. (2018). El Ingeniero, Los Negocios y la Mercadotecnia. Palibrio. Entrevistas sectoriales.

García, D. (2018). Hace falta talento para 4.0: Leopoldo Cedillo. Milenio

Gerbert, P., Lorenz, M., Rüßmann, \&, Waldner, M. (2015). Industry 4.0: The future of productivity and growth in manufacturing industries. Boston Consulting Group. Disponible en: https://on.bcg.com/2MG2aDa

Gobierno de México (2016). La industria automotriz mexicana: situación actual, retos y oportunidades. Disponible en: https://www.gob.mx/se/articulos/la-industriaautomotriz-es-una-de-las-mas-fuertes-de-mexico

Gobierno de México (2018) TLCAN. Resultados de la Modernización del Acuerdo Comercial México, Estados Unidos y Canadá. Recuperado en https://www.gob.mx/tlcan

Hernández, A. (2019). Profesor investigador de la escuela de Ingeniería. Universidad de Monterrey

Hernández, R., Fernández, C. y Baptista, L. (2014). Metodología de la Investigación (6ª Edición). México: McGraw Hill

Klier, T. \& Rubenstein, J. (2016) Mexico's growing role in the auto industry under NAFTA: Who makes what and what goes where. Economic Prospective, 41(6) 
Martinazioli, R. (2019). Coordinador de Comercio Exterior. Clúster Automotriz de Nuevo León

Nava, R (2014). Buscan capacitar empresas Tier 2. Revista somos industria, (2). 10-14 Navarrete-Báez, F \& Sicilia, D. (2016). La industria Automotriz en México: potencia económica para el siglo XXI. Revista de la Universidad del Valle de Atemajac, (86). Disponible en: https://bit.ly/32/UpZH

Ochoa, L. (2019) Coordinador del Comité Tier 2. Clúster Automotríz de Nuevo León

Oxford Business Group (2018). The Report Mexico. Disponible: https://oxfordbusinessgroup.com/mexico-2018

ProMéxico (2015). México Automotive. Revista ProMéxico. (4), 10-15.

ProMéxico (2017). Industry 4.0 The Fourth Industrial Revolution is On. Revista ProMéxico (6), 12-17

Roblek, V., Meško, M., \& Krapež, A. (2016). A complex view of Industry 4.0. SAGE open, 6(2). Disponible en: https://doi.org/10.1177/2158244016653987

Tijerina, H. (2019). Director de Inversión extranjera del Gobierno. Estado de Nuevo León, México

Unger, K., \& Chico, R. (2004). La industria automotriz mexicana en una perspectiva de clusters regionales. El trimestre económico, 4(284). 909-941. Disponible en: https://www.redalyc.org/pdf/313/31328406.pdf

Val, J (2016). Industria 4.0: La transformación digital de la industria. Conferencia de directores y decanos de Ingeniería Informática. Disponible en: http://coddii.org/wp-content/uploads/2016/10/Informe-CODDII-Industria4.0.pdf

Vargas, J. (2016). La industria mexicana de autopartes su evolución y perspectivas ante la globalización. Mercados y Negocios. 9(5). 2-11. Disponible en: http://revistascientificas.udg.mx/index.php/MYN/article/view/4962/4614

Nava, K., Silva, A., Guajardo, A., Leyva U., \& Torres, C. 
Zegarra, C, \& Pérez, M. (2018). Industria 4.0 oportunidades y retos en México. Revista Forbes, México. Disponible en: https://www.forbes.com.mx/industria-4-0oportunidades-y-retos-en-mexicol

Industria 4.0 en el sector de autopartes 